\title{
Eidgenössische Wahlen 2011
}

\author{
Am 23. Oktober 2011 entscheidet das Schweizer Stimmvolk, wer für die kommenden \\ vier Jahre die Politik im Parlament bestimmt. Als wichtiger Akteur im Gesundheitswesen \\ und als Berufsverband mit über 35000 Mitgliedern ist es der FMH ein Anliegen, dass \\ auch die Ärzteschaft in den beiden Räten vertreten ist und die politischen sowie die \\ gesetzgeberischen Prozesse mitgestalten kann. Nachfolgend stellen wir Ihnen die \\ Kandidatinnen und Kandidaten vor, die sich für den National- oder Ständerat bewerben.
}

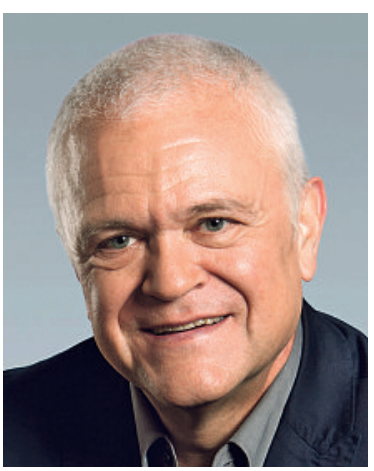

Jürg Knuchel, 1959, Aarau

- Nationalratskandidat SP

- Facharzt FMH für Innere Medizin und Gastroenterologie

- Leitender Arzt für Gastroenterologie und Hepatologie am Kantonsspital Aarau, Einwohnerrat Aarau seit 2009

- Mitglied des kantonalen und Zentralvorstandes des VSAO 1988-1997

- Keine Interessenbindungen

- www.juerg-knuchel.ch

- juerg.knuchel@bluewin.ch
Die zunehmende Bedeutung von Ökonomie und Prozessqualität im Gesundheitswesen erfordert in den nächsten Jahren unsere volle Aufmerksamkeit, damit wir eine Zweiklassenmedizin verhindern und gleichzeitig die zentralen, zutiefst menschlichen Aspekte der Medizin und eine optimale, medizinische Versorgung für alle uneingeschränkt erhalten können. Dafür möchte ich mich auf allen Ebenen einsetzen und damit zum Brückenschlag zwischen Grundversorgern und Spezialisten, zwischen Ökonomie und Medizin beitragen - zum Wohl und zum Vorteil unserer Patienten und damit letztlich von uns allen!

Aargau

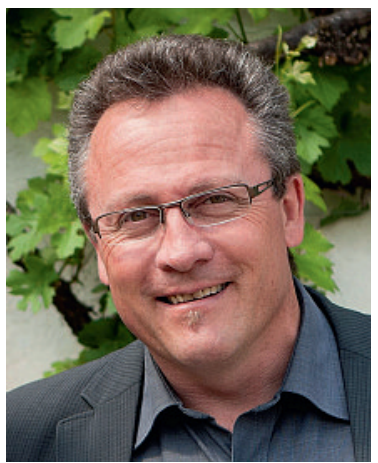

Bern

\section{Daniel Beutler-Hohenberger, 1962,} Mühlethurnen

- Nationalratskandidat EDU

- Allgemeine Medizin FMH, Manuelle Medizin SAMM

- Hausarzt in eigener Praxis, Publizist

- Co-Präsident Dachverband Drogenabstinenz Schweiz, Mitglied Referendumskomitee Teilrevision BetmGes, Mitglied Initiativkomitee Abtreibungsfinanzierung ist Privatsache

- Ehem. Generalsekretär der Arbeitsgemeinschaft Evangelischer Ärzte der Schweiz, AGEAS

- Keine Interessenbindungen

- www.daniel-beutler-hohenberger.ch

- dan.beutler@hin.ch
Für ein bezahlbares Gesundheitswesen, $u$. a. durch Förderung der Hausarztmedizin, für eine vernünftige Gesundheitspolitik mit restriktivem Grundleistungskatalog, für den differenzierten Einsatz medizinischer Leistungen und für mehr Eigenverantwortung.

Für ein umfassendes Lebensrecht am Lebensbeginn und -ende, basierend auf der unteilbaren Würde allen menschlichen Lebens, d.h. einen umfassenden Schutz des ungeborenen Lebens mit klaren Schranken für die Fortpflanzungsmedizin und ein Verbot der aktiven Sterbehilfe und der Suizidbeihilfe.

Für eine abstinenzorientierte Drogenpolitik mit Erhalt und Förderung abstinenzorientierter Therapien und ein nachhaltiges Behandlungskonzept aller Suchtkranken. Gegen die Ausweitung der Substitutionsbehandlungen und gegen jegliche Legalisierungsbestrebungen. 


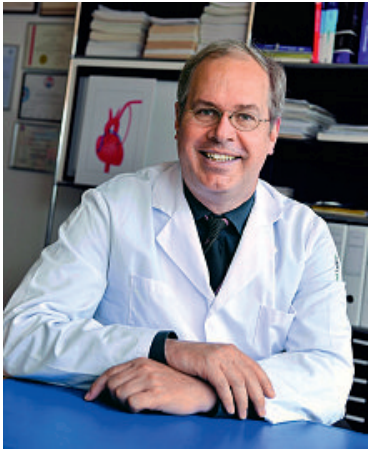

Bern

\section{Thierry P. Carrel, 1960, Bern}

- Nationalratskandidat FDP

- Direktor der Berner Universitätsklinik für Herz- und Gefässchirurgie

_ Allgemeine Chirurgie FMH

- Präsident der Schweiz. Gesellschaft für Herz- und thorakale Gefässchirurgie (2011-2012), Sekretär der Schweiz. Gesellschaft für Thorax-, Herz- und Gefässchirurgie (1996-2010), Vertreter der Gesellschaft in der Ärztekammer und in der KWFB (heute SIWF), Fachvertreter in der Titelkommission

- www.thierry-carrel.ch

- thierry.carrel@insel.ch

Die kantonale Planung soll sich im Spitalbereich auf das Notwendigste beschränken. Es darf auf keinen
Fall eine Staatsmedizin daraus entstehen. Im Gesundheitswesen sollen zukünftig viel mehr Regionen statt Kantone in die Strategie einbezogen werden. Die freie Arzt- und Spitalwahl muss ohne Einschränkungen gewährleistet sein.

Der Numerus clausus muss zwingend gelockert werden. Es fehlen nicht nur Hausärzte, sondern auch zunehmend Spezialärzte. Die Auswahl von zukünftigen Medizinstudenten darf nicht aufgrund von fragwürdigen psychologischen Tests stattfinden. Vielmehr müssen Fachwissen, Motivation, Gesprächsfähigkeit und Empathie berücksichtigt werden.

Viel zu tun gibt es bei der Lösung ethischer Probleme (Prä-Implantationsdiagnostik, Organspende, Sterbehilfe) sowie bei der Aufwertung der Pflegeberufe, ohne sie zu akademisieren.

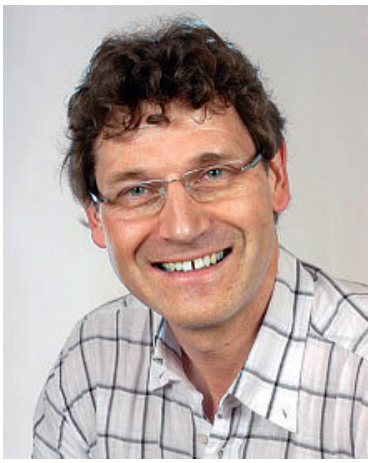

Bern

\section{Willfried Gasser, 1957, Wabern}

- Nationalratskandidat EVP

- Dr. med., kein Facharzttitel

- Consultant, Unternehmensberatung

- Grossrat Kanton Bern von 2005-2010, Mitglied Oberaufsichtskommission

- Präsident Schweizerische Evangelische Allianz

- www.wilfgasser.ch

- wi.gasser@bluewin.ch

Auf nationaler Ebene müssen die richtigen Anreize geschaffen werden für eine qualitativ gute, aber kostenbewusste Gesundheitsversorgung. Die unterschiedliche Finanzierung von ambulant und stationär muss auf- gehoben werden, weil dies unter dem Aspekt einer integrierten Versorgung keinen Sinn macht. Mir liegt an einem fairen («gleich lange Spiesse») und starken Wettbewerb zwischen privaten und öffentlichen Spitälern. Den Wettbewerb unter den Krankenkassen erachte ich jedoch als wenig hilfreich für die Kostendämpfung und unterstütze die Idee einer Einheitskasse.

Kantonal setzte ich mich 2010 mit einer Motion für einen Neubau des Stadtspitals auf dem Inselareal ein. Eine Strukturbereinigung ist nötig, und dazu braucht es eine klare kantonale Planung. Das Spitalversorgungsgesetz muss mittelfristig durch ein Gesundheitsversorgungsgesetz ersetzt werden.

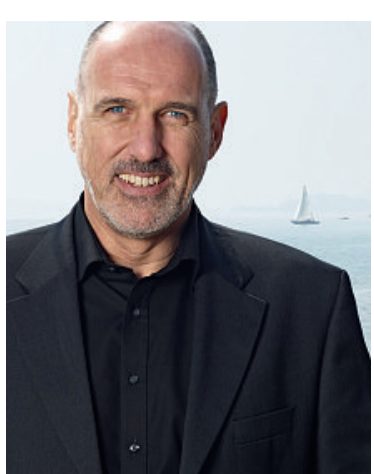

\section{Jacques de Haller, 1952, Bern}

- Nationalratskandidat SP

- Allgemeinmedizin FMH

- Präsident der FMH

- Präsident der SGAM (1997-2004); verschiedene andere standespolitische Mandate

- Keine Interessenbindungen

- www.jacquesdehaller.ch

- jdh@jacquesdehaller.ch, jdh@fmh.ch
Ich kandidiere bei den Parlamentswahlen 2011, weil ich meine Erfahrungen als Arzt und Präsident der FMH als Nationalrat in die Politik einbringen möchte. Die politischen Anliegen der Ärztinnen und Ärzte sind mir bestens vertraut. Durch meine Präsenz unmittelbar dort, wo die Entscheidungen getroffen werden, und mein mittlerweile dichtes und breitgefächertes Netzwerk in der Gesundheitspolitik will ich der FMH entscheidende Vorteile bringen.

Unser Gesundheitssystem soll gerecht, effizient und allen zugänglich sein - jede und jeder soll von qualitativ hochstehenden medizinischen Leistungen profitieren können. Dazu braucht es gutausgebildete und genügend Ärztinnen, Ärzte und Pflegefachpersonen, die unter optimalen Arbeitsbedingungen agieren können.

Dafür will ich mich politisch engagieren.

\section{Bern}




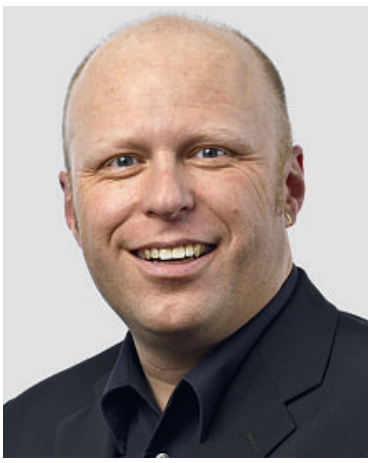

Bern

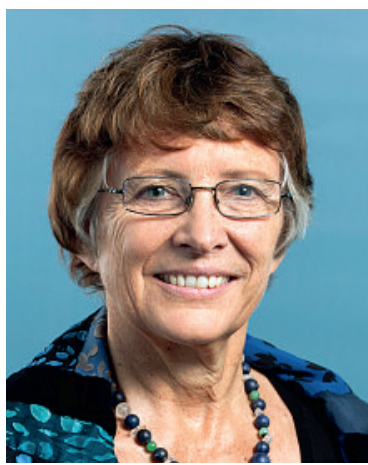

Bern
Dieter Hötsch,1964, Urtenen-Schönbühl

- Nationalratskandidat glp

- Orthopädische Chirurgie FMH

- Versicherungsmedizinischer Berater und Gutachter

- Mitglied Honorar- und Tarifkommission der SGOT seit 2002

- Keine Interessenbindungen

- www.be.grunliberale.ch

- hoetsch@intergga.ch

In meiner Funktion als Versicherungsmediziner aus einem operativen Fachgebiet muss ich bei den Kontakten mit verschiedensten Playern in unserem
Gesundheitswesen leider immer wieder erkennen, dass beim Verständnis füreinander noch grosse Defizite vorhanden sind, nicht zuletzt unter den verschiedenen Disziplinen unserer Gilde. Mein wichtigstes gesundheitspolitisches Ziel ist es deshalb, im Interesse einer hochstehenden Versorgung unserer Bevölkerung die verschiedenen Gruppen an einen Tisch zu bringen, wo in konstruktiver Atmosphäre die anstehenden Probleme erörtert und tragfähige Lösungen gefunden werden. Ich werde mich dafür einsetzen, dass wir Ärztinnen und Ärzte wieder als kompetente Partner wahrgenommen werden, die konsensorientiert an der Zukunft unseres Gesundheits- und Sozialwesens mitarbeiten.

\section{Danielle Lemann, 1950, Langnau}

- Nationalratskandidatin SP

- Allgemeine Medizin FMH

- Hausärztin in Gemeinschaftspraxis, Teilzeitärztin am Regionalspital Emmental

- Grossrätin Kanton Bern

- Expertin für Komplementärmedizin Eidgenössische Arzneimittelkommission, Delegierte Bernische Ärztegesellschaft, Vorstand; Anthroposophisch Orientierte Ärzte in der Schweiz

- Interessenbindungen: Ärzte für Umweltschutz, SAC, Solidarmed, PSR

- www.danielle-lemann.ch

- dlemann@hin.ch

- Ich setze mich ein für eine echte Stärkung der hausärztlichen Grundversorgung: endlich gerechte TARMED- Positionen für die Grundversorger, Vermeidung von leeren Worthülsen wie
Managed Care, aber weitere Förderung der Vernetzung der Teilnehmer im Gesundheitswesen, mehr Hausarztprofessorinnen und -professoren.

- Achtsame Einführung der Fallpauschalen erst nach Regelung der noch ungelösten Probleme, wie Datenschutz, Aus- und Weiterbildung der Ärztinnen und Ärzte, Behandlung von polymorbiden, palliativen und pädiatrischen Patientinnen und Patienten, Berücksichtigung des Pflegeaufwandes.

- Umsetzung des neuen Verfassungsartikels Ja zur Komplementärmedizin mit den fünf Grundforderungen.

- Umsetzung der Anliegen der Ärztinnen und Ärzte für Umweltschutz und Ärztinnen und Ärzte für soziale Verantwortung.

- Förderung der Palliativmedizin und des Bewusstseins unserer Endlichkeit.

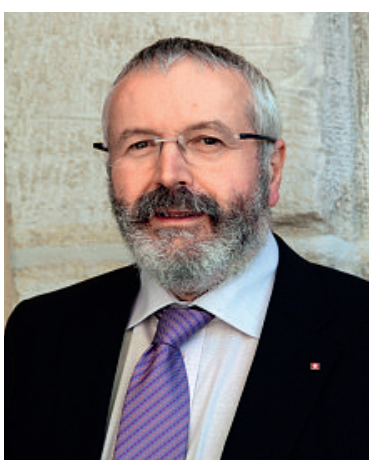

Jura

\section{Dominique Baettig, 1953, Delémont}

- Candidat pour le Conseil national UDC

- Psychiatrie FMH

- Pratique libérale

- Conseil national (2007-2011), Conseiller de Ville Delémont (2004-2012)

- Aucun lien d'intérêts

- www.udc-jura.ch

_ dominiqueb@bluewin.ch

Aujourd'hui, l'enjeu politique n'est plus entre une gauche sociale et une droite économique, mais entre les partisans et les résistants à la mondialisation.
Entre une oligarchie financière et déracinée et les citoyens enracinés, rattachés à une culture, une identité, à la démocratie directe de proximité, dans une entité de taille humaine et historique. L'UDC incarne ces valeurs de résistance morale, spirituelle et d'esprit d'entreprise, de passion de libertés.

En tant que médecin attaché aux valeurs non marchandes, je m'inquiète des dérives de la médecine technique, industrielle, de redistribution illusoire et coûteuse d'un capital de soins de santé. Le médecin doit rester un conseiller de confiance, soucieux de ne pas nuire. Les soins gérés sont une dérive de gestion économique dangereuse. 


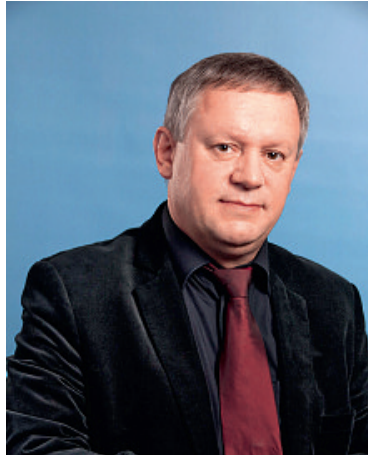

Pierre-Alain Fridez, 1957, Fontenais

- Candidat pour le Conseil national PS

- Médecine générale FMH

- Cabinet médical à Fontenais

- Député, ancien maire

- Membre commission politique de santé MFE

- pafmabe@bluewin.ch

Médecin généraliste installé dans un village en Ajoie depuis 23 ans, j'ai assumé en parallèle depuis 15 ans différents mandats politiques, en particulier maire de ma commune durant 12 ans et député pendant 9 ans Jura au sein du Parlement jurassien.
Mes motivations pour briguer un siège au Conseil national résident bien entendu dans la défense des valeurs qui m'ont fait adhérer au parti socialiste: justice et protection sociale, défense de l'environnement, égalité des chances notamment, mais également dans mon engagement prioritaire dans le domaine de la politique de la santé: combat en faveur d'une caisse maladie unique, reconnaissance et renforcement du statut des médecins de premier recours, prévention et promotion de la santé, égalité d'accès aux soins.

Je siège au sein de la Commission politique de l'association suisse des médecins de famille MFE.

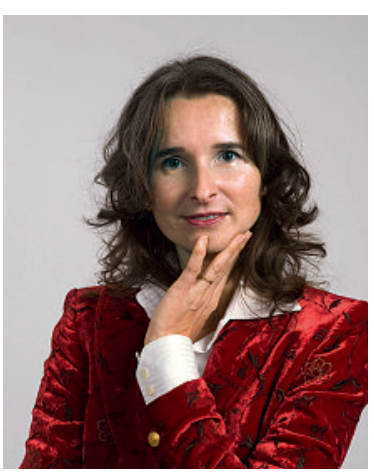

Luzern

\section{Yvette Estermann, 1967, Kriens}

- Nationalratskandidatin SVP

- Dr. med., Zusatz-Ausbildung in klass. Homöopathie bei SVHA und SAHP

- Beraterin in eigener Firma: «Estermann life coaching»

- Kantonsrätin 2005-2007, Nationalrätin ab 2007, Mitglied der Kommission für soziale Sicherheit und Gesundheit NR (SGK-NR)

- Mitglied bei VSAO und SAHP

- www.yvette-estermann.ch und www.estermannaktuell.ch

- info@yvette-estermann.ch
Ob früher als Kantonsrätin, Präsidentin der Gesundheitskommission im kantonalen Parlament oder jetzt auch als Nationalrätin und Mitglied der SGK-NR setze ich mich für ein Gesundheitssystem ein, welches den Bedürfnissen unserer Bevölkerung entspricht. Dies muss aber bezahlbar sein und möglichst frei von Missbrauchsmöglichkeiten. Als Ärztin weiss ich, was für eine wichtige und verantwortungsvolle Aufgabe in unserer Gesellschaft die Ärztinnen und Ärzte erfüllen. In diesem Sinne habe ich immer bei politischen Anliegen gehandelt und gedenke, dies auch weiterhin zu tun. Ich bin überzeugt, dass alle Ärztinnen und Ärzte sowie ihre Mitarbeiter im Gesundheitssektor immer nach ihrem besten Wissen und Gewissen handeln, - im Dienst der Bevölkerung. Diese Bemühungen unterstütze ich gern, - mit Überzeugung!

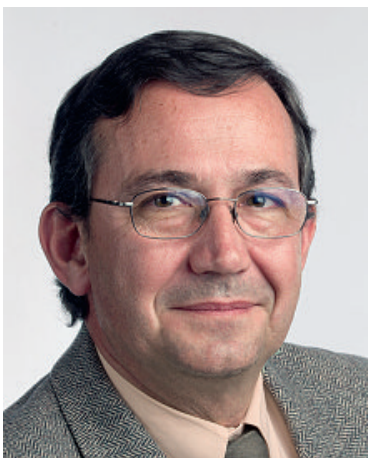

\section{Blaise Courvoisier, 1955, La Chaux-de-} Fonds

- Candidat pour le Conseil des Etats UDC

- Chirurgie FMH

- Indépendant en cabinet et clinique privée

- Député au Grand Conseil neuchâtelois,

- Président commission santé du Grand Conseil

- Président du Conseil d'Administration Clinique de la Tour

- www.udc.ne

- b.courvoisier@bluewin.ch
La santé et son enseignement doivent être régionalisés, au détriment de la cantonalisation actuelle. Il faut avoir un réseau d'hôpitaux régionaux de proximité pour les soins de base et des centres universitaires régionalisés et redimensionnés pour les pathologies les plus graves. La médecine doit être enseignée dans ces centres régionaux avec la possibilité pour un nombre restreint d'étudiants de poursuivre en milieu universitaire.

\section{Neuchâtel}




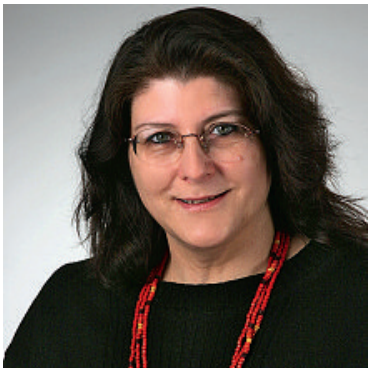

St. Gallen
Veronika Breitler-Voigt, 1957, Kirchberg

- Nationalratskandidatin BDP

- Dr. med., Dipl.-Psych., FMH Psychiatrie/Psychotherapie, Fachärztin für Psychosomatische Medizin, SAPPM Psychosoziale Medizin, Systemische Familientherapie, Psychoanalyse, Gruppenanalyse SGAZ (Zürich und London)

- Niedergelassen in eigener Praxis

- Keine Interessenbindungen

- www.ostschweizerinnen.c und www.bdp-sg.ch

- www.drbreitler.ch

- veronika.breitler@tbwil.ch
Dafür engagiere ich mich in der BDP:

- Gesundheitspolitik (u.a. mehr Eigenverantwortung der Bevölkerung für die eigene Gesundheit);

- Sozialwerke (sinnvolle Wiedereingliederung nach IV);

- Frauenfragen (Gender, Vereinbarkeit von Karriere und Lifebalance);

- Familienpolitik, Bildung, öffentliche Sicherheit.

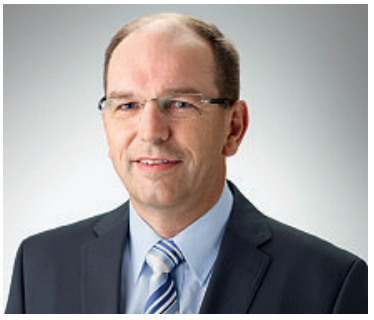

\section{Bruno Damann, 1957, Gossau}

- Nationalratskandidat CVP

- Allgemeinmedizin FMH

_ Praxistätigkeit im Ärztehaus Gossau

_ Parlamentarier der Stadt Gossau

- Interessenbindungen: Adviser $\mathrm{HCl}$ Galenica

- www.Bruno-Damann.ch

- bdamann@hin.ch, Bruno.Damann@bigfoot.com
Ich vertrete die freie Arzttätigkeit. Für mich ist es wichtig, dass die Grundversorger gestärkt werden. Freier Zugang zur Medizin für alle. Der staatliche Einfluss auf die Medizin darf nicht noch mehr zunehmen. Hausarztmodelle ausbauen. Spitalplanung über die Kantonsgrenze hinaus. Keine ambulanten Fallpauschalen.

\section{St. Gallen}

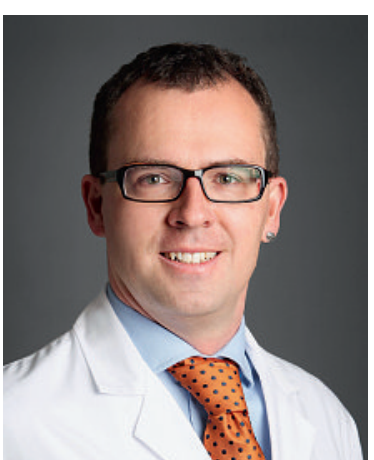

St. Gallen

\section{Friedrich von Toggenburg, 1974, Buchs}

- Nationalratskandidat CVP

- Urologie / operative Urologie

- Praxisarzt

- Keine Interessenbindungen

- www.friedrich-vontoggenburg.ch

- friedrich.vontoggenburg@hin.ch

Die Einführung der DRG ist beschlossen und kann, realistisch betrachtet, nicht mehr aufgeschoben werden. Um die negativen Auswirkungen dieses Systemwechsels möglichst bald zu eliminieren, ist eine intensive Begleitforschung notwendig. Dies muss insbesondere auch von parlamentarischer Seite gefordert werden.

Die Problematik der unzureichenden Anzahl medizinischer Ausbildungsplätze muss schnell gelöst werden. Wir können uns nicht erlauben, dass weiterhin mehr als ein Viertel der benötigten Ärzte aus dem Ausland angeworben werden müssen.

Der stetige Anstieg der OKP-Prämien kann langfristig nur durch eine Straffung des OKP-Kataloges gebremst werden. Dieses ethisch wie politisch schwierige Thema muss bald, aber breit abgestützt angegangen werden.

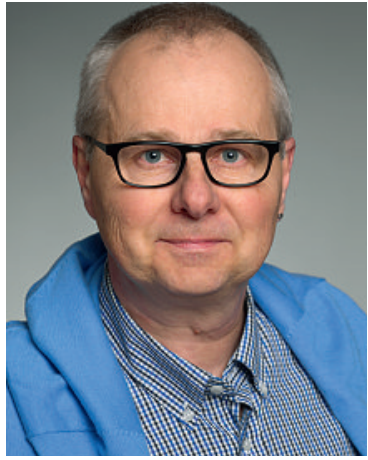

Thurgau

\section{Bernhard Wälti, 1956, Freidorf}

- Nationalratskandidat SP

- Allgemeinmedizin FMH

- Selbständig als Landarzt

- Kantonsrat Thurgau seit 2005

- ehem. Vorstandsmitglied VSAO St. Gallen; seit 1995 Lehrbetrieb für MPA

- Präsident Energiegenossenschaft Roggwil, Beirat Thurgauer Programm zur Brustkrebsfrüherkennung, Mitglied Ärztegesellschaft Thurgau, Mitglied Pro Natura Thurgau

- www.worthalten.ch

- bmwaelti@hin.ch
Die bizarre Bürokratie muss fallen!

Andere haben ihr Gesundheitswesen ruiniert. Lernen wir von ihnen und machen wir nicht die gleichen Fehler! Unser erstklassiges Gesundheitswesen darf nicht von Leuten zerschlagen werden, die davon nichts verstehen, und ohne dass wir Besseres zur Verfügung haben. Deshalb brauchen wir mehr Fachwissen und Unvoreingenommenheit in der Politik, nicht Ideologie. Denn Medizin kennt keine Partei, sondern Vernunft.

Wenn wir die Stärke der Schweiz bewahren wollen, müssen wir ihren Zusammenhalt erhalten. Dazu gehört, dass Medizin bezahlbar ist. Wir Ärzte - Hausärzte wie Spezialisten - leisten erstklassige Arbeit. Dafür wollen wir faire Bedingungen, keine bizarre Bürokratie. Und wir wollen Patienten mit Eigenverantwortung, gezielte Forschung, ausreichend Studienplätze. Eine clevere Medizin eben. 


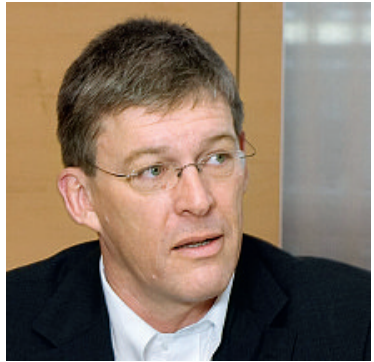

Thurgau

\section{Mathias Wenger, 1956, Häuslenen}

- Nationalratskandidat CVP

- FMH Innere Medizin

- Kantonsarzt Kt. Thurgau zu 85\%,

Vertrauensarzt Bundesamt für Zivilluftfahrt und Bundesamt für Verkehr zu 15\% (1991-2007

Hausarzt; 2007-2010 teilzeitlich Geschäftsführer Schlossberg Ärztezentrum Frauenfeld, kleines Pensum als Hausarzt und Vertrauensarzt, 50\% stellvertretender Kantonsarzt Thurgau)

- 1986-1988: Vizepräsident VSAO, Sektion Thurgau, 1992-2000 Vorstandsmitglied Ärztegesellschaft Thurgau, 2000-2005: Präsident Ärztegesellschaft Thurgau, 2004-2005 Co-Präsident VEDAG, seit 2005 bis heute Vorstandsmitglied VEDAG, 2008 bis 2010: Vorstandmitglied SGARM

- Interessenbindungen: Vorstandsmitglied VEDAG, Kantonsarzt, Vertrauensarzt BAZL, BAV

- www.mathias.wenger.ch

- mathias.wenger@hin.ch
Dafür engagiere ich mich:

- Chancengerechte, medizinische Versorgung und Finanzierung, d. h. Eigenverantwortung, Solidarität, Subsidiarität;

- Vernetzung zwischen Grundversorgern, Spezialisten und Spitälern über die gesamte Behandlungskette, mit Einbezug der Pflege und des Sozialwesens;

- Förderung des Nachwuchses in Pflege und ärztlichem Bereich;

- Förderung der Grundversorgung, der psychischen Gesundheit der Prävention und der Gesundheitsförderung;

- Kostenträger sind keine Leistungsanbieter;

- Korrekte Qualitätsmessung, faire Wirtschaftlichkeitskontrollen, direkte Medikamentenabgabe, adäquate, nicht kantonsabhängige Leistungsabgeltung.

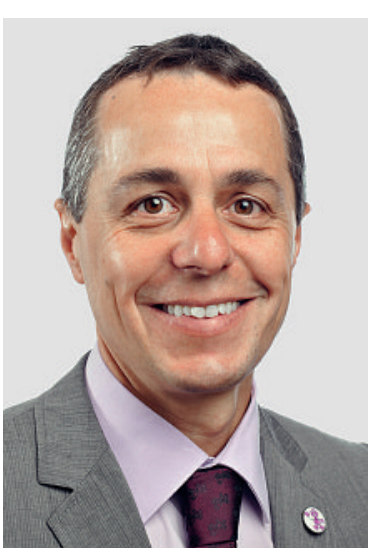

Ticino

\section{Ignazio Cassis, 1961, Collina d'Oro}

- Candidato al Consiglio nazionale PLR

- FMH prevenzione e salute pubblica, $\mathrm{FMH}$ medicina interna

- Consigliere Nazionale e Vicepresidente della FMH

- Dal giugno 2007 membro del Consiglio nazionale, dall'aprile 2004 membro del Consiglio comunale

- Dal giugno 2008 Vicepresidente della FMH, 1992-1996 Vicepresidente ASMAC; 1989-1992 Presidente ASMACT

- Presidente della Fondazione EQUAM, Presidente di Fourchette verte Ticino, Membro di comitato del Forum Managed Care

- www.ignaziocassis.ch

- ignazio.cassis@parl.ch
Nella legislatura 2007-11 mi sono impegnato in Parlamento per una nuova Legge federale sulla prevenzione e per migliorare la LAMal. Ho proposto - con successo - una soluzione per impedire alle casse malati di selezionare le persone sane. Mi sono battuto per migliorare il modo di calcolare l'economicità dei medici e per modellare due grosse riforme strutturali: quella sul finanziamento ospedaliero e quella sulle cure integrate. Nella FMH ho stimolato il lavoro in Comitato e negli altri Organi. Sono stato ospite di numerose società mediche cantonali e specialistiche per spiegare il lavoro politico e la sua logica. Desidero essere rieletto in Parlamento perché vorrei proseguire il lavoro proprio ora che ho maggior competenza e legittimazione. Perciò mi permetto di chiedere il vostro sostegno.

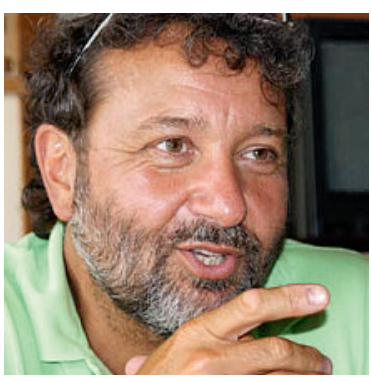

Ticino

\section{Paolo Cipriano Emanuele Peduzzi, 1963, Bellinzona}

- Candidato al Consiglio nazionale PPD

- Pediatra

- Studio medico in proprio a Bellinzona e Medico Scolastico

- Gran Consigliere in carica dal 2010

- Presidente del Trust Center Ticino

- Conflitti d'interessi: Presidente di alcune Associazioni caritative senza lucro

- www.ilpediatra.ch - www.pesovia.ch - www. Con-Tatto.ch

- paolo@ilpediatra.ch
Nato in Ticino da una famiglia che si è sempre interessata di ciò che succede attorno, ho sempre abbinato il mio studio, la mia formazione e ora la mia attività professionale con attività politiche. La politica è un modo di mettersi a servizio degli altri e della comunità. Come nella parabola dei talenti: chi ne ha li metta a disposizione!

La politica sanitaria svizzera è stata stravolta in questi anni e come medici siamo spettatori ignari di capovolgimenti tali nella politica sanitaria da mettere a repentaglio l'arte medica tout-court! Una nostra presa di posizione e una nostra attiva partecipazione alle decisioni di politica sanitaria federale e cantonale non può essere procrastinata, ne va della qualità della medicina stessa e della salute in senso più largo della nostra Nazione e della nostra gente. 


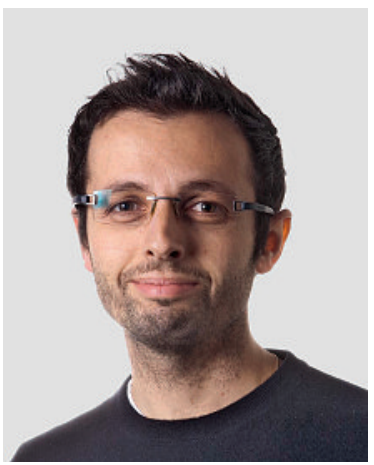

Zürich

\section{David Garcia Nuñez, 1975, Zürich}

- Nationalratskandidat AL

- Psychiatrie und Psychotherapie

- Oberarzt in der Klinik für Psychiatrie und Psychotherapie am UniversitätsSpital Zürich

- Ex-Mitglied der Ständigen Kommission für Weiterund Fortbildungsfragen der SGPP

- Keine Interessenbindungen

- dagar@doctor.com

DRG/Fallpauschalen

- Sofortiges Moratorium, bis Fragen der Begleitforschung, der med. Aus- und Weiterbildung und der Sicherstellung genügend qualifizierter med. Stellen geklärt sind
Stärkung der medizinischen Grundversorgung

- Förderung von Massnahmen, um die qualitativ hausärztliche und psychiatrische Betreuung der Bevölkerung zu gewährleisten.

Besserstellung nicht-invasiver Leistungen

- Um «medizinischen Aktionismus» möglichst zu unterbinden, sollte die Entgeltungskluft zwischen invasiven und nicht-invasiven Interventionen möglichst reduziert werden.

Abschaffung des Numerus clausus

- Notwendige Ressourcen, um genügend Medizinerinnen und Mediziner auszubilden, werden zur Verfügung gestellt. Nur so kann die sich bereits abzeichnende katastrophale Versorgungssituation abgewendet werden.

Einführung einer einkommensabhängigen Krankenkassenfinanzierung.

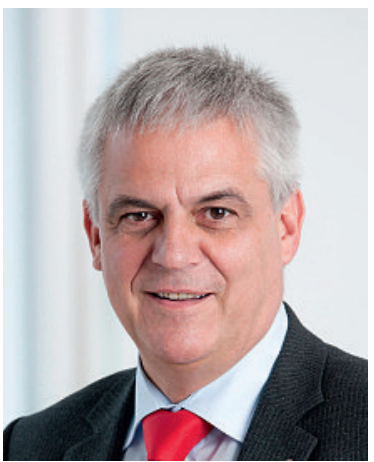

Josef Widler, 1954, Zürich

- Nationsratskandidat CVP

- Allgemeine Medizin FMH

- Hausarzt, Ärztlicher Leiter Ärztehaus Zürich West

- Gemeinderat der Stadt Zürich

- Vizepräsident der Ärztegesellschaft Kanton Zürich

- Keine Interessenbindungen

- www.josef-widler.ch

- josef.widler@bluewin.ch
Die Kosten unseres Gesundheitswesens werden durch strukturelle Mängel unnötig in die Höhe getrieben.

Deshalb setze ich mich ein für

- die Aufhebung des Kontrahierungszwanges,

- die Aufhebung des Tarifschutzes,

- die Vereinfachung des Kosten treibenden Versicherungssystems (KGV, UVG, IV),

- die Einführung einer Einheitskrankenkassenprämie,

- die Abschaffung nicht evidenzbasierter Qualitätskontrollen,

- die Straffung des Leistungskataloges,

- die Zentralisierung der Spitzenmedizin.

\section{Zürich}

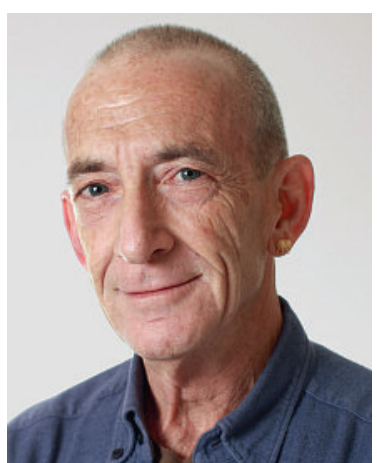

Zürich

\section{David Winizki, 1948, Zürich}

- Nationalratskandidat AL

- Allgemeine Medizin FMH

- Hausarzt

- Keine Interessenbindungen

- david.winizki@hin.ch

Als Hausarzt werde ich oft konfrontiert mit der Verarmung breiter Bevölkerungsschichten, immer knauserigeren Sozialversicherungen, omnipotenteren Krankenkassen und einer zunehmenden Ökonomisierung des Gesundheitswesens. Dieses gehört jedoch zu jenen Gütern, die wir nicht zum Spass konsumieren, sondern zum Überleben brauchen. Es muss deshalb der ganzen Bevölkerung ohne Rationierungen zugänglich sein und solidarischer als heute bezahlt werden.

Budgetverantwortung oder Fallpauschalen implizieren Rationierungen für Grundversicherte und sind deshalb ungerecht. Leistungserbringer sollen gut aus-, weiter- und fortgebildet werden, vernetzt arbeiten und adäquat bezahlt werden. Existenzbedingungen, die ein selbstbestimmtes Leben in Würde erlauben, sind als Gesundheitsvorsorge wichtiger als Birchermüesli und Joggen. 